\title{
Wavelet Interpolation Method for Solving Singular Integral Equations
}

\author{
Yousef Al-Jarrah, En-Bing Lin \\ Central Michigan University, Mt. Pleasant, USA \\ Email: enbing.lin@cmich.edu
}

Received July 24, 2013; revised August 24, 2013; accepted August 31, 2013

Copyright (C) 2013 Yousef Al-Jarrah, En-Bing Lin. This is an open access article distributed under the Creative Commons Attribution License, which permits unrestricted use, distribution, and reproduction in any medium, provided the original work is properly cited.

\begin{abstract}
Numerical solutions of singular Fredholm integral equations of the second kind are solved by using Coiflet interpolation method. Error analysis of the method is obtained and examples are presented. It turns out that our method provides better accuracy than other methods.
\end{abstract}

Keywords: Singular Fredholm Integral Equation; Coiflet; Wavelet; Lipschitz Condition

\section{Introduction}

In the early 1900s, Ivar Fredholm solved the integral equations named after him,

$$
y(x)=g(x)+\int_{a}^{b} k(x, t) y(t) \mathrm{d} t,
$$

where the function $g(x)$ and continuous kernel $k(x, t)$ are given, and the unknown function $y(x)$ is to be determined. A numerical method of solving this equation has been shown in [1]. In this study, we discuss the numerical solution of singular Fredholm integral equation of the second kind which is defined as follows:

$$
\begin{aligned}
u(x)= & f(x)-\int_{a}^{b} k(x, t)|x-t|^{\alpha} u(t) \mathrm{d} t, \\
& -1<\alpha<0, a \leq x \leq b,
\end{aligned}
$$

where the functions $f(x)$ and $k(x, t)$ are given, the numerical solution for Equation (1) is to provide an approximation for the unknown function $u(x)$. In fact, Equation (1) is known as an Abel's integral equation which is defined by Niels Henrik Abel. There are many approaches to find a numerical solution of the Abel's equation [2], such as Gauss-Jacobi quadrature rule which was proposed by Fettis (1964), orthogonal polynomials expansion by Kosarev (1973), the Chebyshev polynomials of the first kind by Piessens and Verbaeten (1973) and Piessens (2000), etc. Recently, K. Maleknejad, M. Nosrati and E. Najafi solved the equation by using wavelet Galerkin method [3]. Here we used Coiflets to find a numerical solution of Equation (1).
The Coiflets are discussed in the next section briefly. In Section 3, we solve Abel's Equation (1) by using Coiflets. The error analysis is discussed in Section 4. Finally, we apply our method for two singular equations in the examples and compare our method with other method [3]. We obtain numerical solutions which have achieved better accuracy.

\section{Coiflets and Wavelet Interpolation}

In the context of wavelet theory, we usually deal with wavelets and scaling functions [4]. The wavelet function is defined by building a sequence upon scaling functions generated by $\varphi(x)$. Choosing some suitable sequence, $\left\{a_{p}, p \in Z\right\}$, we obtain the following dilation equation,

$$
\varphi(x)=\sum_{p} a_{p} \varphi\left(2^{j} x-p\right)=\sum_{p} a_{p} \varphi_{j, p}(x)
$$

A nested of subspaces $\left\{V_{j}, j \in Z\right\}$ of $L^{2}(R)$ is defined such that,

$$
V_{j}=\overline{\operatorname{Span}\left\{\varphi_{j, p}(x)\right\}_{p}}, j \in Z
$$

which means that for any function $f(x) \in V_{j}$ it can be expressed as:

$$
f(x)=\sum_{p} \alpha_{p} \varphi_{j, p}(x)
$$

If the basis functions of a subspace are orthogonal at the same level, then a given function $f(x) \in V_{j}$ can be expressed as follows: 


$$
f(x)=\sum_{p}\left\langle f, \varphi_{j, p}\right\rangle \varphi_{j, p}(x)\left\langle f, \varphi_{j, p}\right\rangle
$$

where

$$
\left\langle f, \varphi_{j, p}\right\rangle=\int_{-\infty}^{\infty} f(x) \varphi_{j, p}(x) \mathrm{d} x
$$

If the nested sequence of the subspaces $\left\{V_{j}, j \in Z\right\}$ has the following properties then it is called a multiresolution analysis (MRA):

1) $V_{j} \subset V_{j+1}$

2) $\bigcap_{j \in Z} V_{j}=\{0\}$

3) $\overline{\bigcap_{j \in Z} V_{j}}=L^{2}(R)$

4) $f(x) \in V_{n} \Leftrightarrow f(2 x) \in V_{n+1}$

5) there exists a function $\varphi \in V_{0}$ such that $\{\varphi(x-k), k \in Z\}$ is an orthogonal basis for $V_{0}$.

The wavelet function is constructed in the orthogonal complement of each subspace $V_{j}$ in $V_{j+1}$ which is denoted by $W_{j}$. This means $V_{j+1}=V_{j} \oplus W_{j}$. Since

$$
V_{j} \rightarrow \begin{cases}0, & \text { as } j \rightarrow-\infty \\ L^{2}(R), & \text { as } j \rightarrow \infty\end{cases}
$$

we have $V_{j+1}=V_{j} \oplus W_{j}$ and $L^{2}(R)=\bigoplus_{j=-\infty}^{\infty} W_{j}$. The set $\left\{\psi_{j, p}(x)=\psi\left(2^{j} x-p\right)\right\}$ forms a basis for $W_{j}$, and can be obtained from the following equation:

$$
\psi(x)=\sum_{p} b_{p} \varphi_{j, p}(x), \text { for some } b .
$$

The orthogonally of $W_{j}$ on $V_{j}$ means that any member of $V_{j}$ is orthogonal to the members of $W_{j}$, that is,

$$
\left\langle\varphi_{j, p}, \psi_{j, k}\right\rangle=\int \varphi_{j, p}(x) \psi_{j, k}(x) \mathrm{d} x=\delta_{p, k}
$$

In fact, scaling function and wavelet have the following properties:

$$
\begin{aligned}
& \int \varphi(x) \mathrm{d} x=1 \\
& \int x \varphi(x) \mathrm{d} x=\frac{1}{2} \sum_{p} p a_{p}=C \\
& \int x^{r} \psi(x) \mathrm{d} x=0, r=0, \cdots, N-1
\end{aligned}
$$

where $[0, N]$ is the compact support of $\varphi(x)$ and $\psi(x)$

In what follows, we will recall a scaling function interpolation theorem and the definition of Coiflets. As an application, we will use Coiflets and this interpolation formula to find numerical solutions of singular integral equations.

Definition 2.1. The Coifman wavelet system (Coiflet) of order $L$ is an orthogonal multiresolution wavelet system with

$$
\begin{aligned}
& \int x^{k} \varphi(x) \mathrm{d} x=0, \text { for } k=1,2, \cdots, L-1 \\
& \int x^{k} \psi(x) \mathrm{d} x=0, \text { for } k=0,1, \cdots, L-1
\end{aligned}
$$

Lin and Zhou proved the following interpolation theorem in $R^{2}$ and $R^{n}$ :

Theorem 2.1. [5] Assume the function $(x) \in C^{k}(\bar{\Omega})$, where $\Omega$ is a bounded open set in $\Omega, k \geq N \geq 2$ Let, for $j \in Z$,

$$
\begin{aligned}
& f^{j}(x, y) \\
& =\frac{1}{2} \sum_{p, q \in Z} f\left(\frac{p+c}{2^{j}}, \frac{q+c}{2^{j}}\right) \varphi_{j, p}(x) \varphi_{j, q}(y),(x, y) \in \Omega
\end{aligned}
$$

where the index

$$
\Lambda=\left\{(p, q) \mid\left(\sup \left(\varphi_{j, p}\right) \otimes \sup \left(\varphi_{j, q}\right)\right) \cap \Omega \neq \varphi\right\}
$$

and sup denotes the support of the function.

In addition the moments $M_{l}$ satisfy

$$
M_{j}=\int x^{l} \varphi(x) \mathrm{d} x=c^{l}, l=1,2, \cdots, N-1
$$

Then

$$
\left\|f-f^{j}\right\|_{L^{2}(\Omega)} \leq C\left\|f^{(N)}\right\|_{\infty}\left(\frac{1}{2^{j}}\right)^{N-1}
$$

where $C$ is a constant depending only on $N$ and diameter of $\Omega$;

$$
\left\|f^{(N)}\right\|_{\infty}=\max _{(x, y) \in \Omega ., m=0,1, \cdots, N}\left|\frac{\partial^{N} f}{\partial x^{m} \partial y^{N-m}}(x, y)\right| .
$$

\section{Solving Singular Fredholm Integral Equation Using Coiflets}

This section provides a method of finding numerical solution of Equation (1). In what follows, we assume that $(x, t) \in[a, b] \times[a, b]$ and $k[x, t]$ satisfies Lipschitz condition. The unknown function $u(x)$ in Equation (1) can be expressed in term of scaling functions in the subspacev, where the function $u(x)$ is approximated by $u^{\wedge}(x)$ such that;

$$
u^{\wedge}(x)=\sum_{p} a_{p} \varphi_{j, p}(x)
$$

To find the numerical solution we need to determinate the unknowns $a_{p}$ in Equation (3).

By substituting Equation (3) in (1) we have the following equation,

$$
\sum_{p} a_{p} \varphi_{j, p}(x)+\int_{0}^{1} k(x, t)|x-t|^{\alpha} \sum_{p} a_{p} \varphi_{j, p}(t) \mathrm{d} t=f(x)
$$

which is equivalent to the equation,

$$
\sum_{p} a_{p}\left(\varphi_{j, p}(x)+\int_{0}^{1} k(x, t)|x-t|^{\alpha} \varphi_{j, p}(t) \mathrm{d} t\right)=f(x)
$$


By providing sufficient collocation points in $[0,1]$ for Equation (4) we will have a linear system of linear equations with unknown $a_{p}$. In fact, the linear system can be written as the following matrix equation,

$$
\boldsymbol{a} \cdot A=\boldsymbol{f}
$$

where $\boldsymbol{a}=\left(a_{1}, a_{2}, \cdots, a_{n}\right)$,

$$
\begin{aligned}
\boldsymbol{f}=\left(f\left(x_{1}\right), f\left(x_{2}\right), \cdots, f\left(x_{n}\right)\right) & \text { and } \\
A & =\left[\begin{array}{cccc}
A_{1}\left(x_{1}\right) & A_{2}\left(x_{1}\right) & \cdots & A_{n}\left(x_{n}\right) \\
A_{1}\left(x_{2}\right) & A_{2}\left(x_{2}\right) & \cdots & A_{n}\left(x_{2}\right) \\
\vdots & \vdots & \ddots & \vdots \\
A_{1}\left(x_{n}\right) & A_{2}\left(x_{n}\right) & \cdots & A_{n}\left(x_{n}\right)
\end{array}\right]
\end{aligned}
$$

is obtained from the left hand side of Equation (4). Subsequently, we substitute the solutions of $a_{p}$ into Equation (3), and obtain an approximate solution of the integral equation.

\section{Error Analysis}

The integral Equation (1) can be rewritten as follows [3].

$$
\int_{0}^{1} k(x, t)|x-t|^{\alpha} u(t) \mathrm{d} t=\int_{0}^{1} H(x, t) u(t) \mathrm{d} t
$$

where

$$
H(x, t)= \begin{cases}k(x, t)|x-t|^{\alpha} & x \neq t \\ 0 & x=t\end{cases}
$$

Then the integral Equation (1) is equivalent to the following equation,

$$
u(x)=f(x)-\int_{0}^{1} H(x, t) u(t) \mathrm{d} t
$$

The next theorem shows the convergence rate of our method for solving Equation (1). Without loss of generality, we suppose that the integral equation is defined on the interval $[0,1]$.

Theorem 4.1. In Equation (1), suppose that the function $k$ satisfies the Lipchitz condition. Moreover, $f(x)$ is continuous on the interval $[0,1]$. For $j \in Z$,

$$
u^{j}(x)=\sum_{p} a_{p} \varphi_{j, p}(x)
$$

is an approximate solution of the unknown function in Equation (1) with coefficients obtained in Section 3. Then

$$
\|e(x)\|=\left\|u(x)-u^{j}(x)\right\| \leq c\left(\frac{1}{2}\right)^{j},
$$

for some constant $c$.

Proof: We prove in two cases, one at singularities (case 1) and the other at the points $x \neq t$ (case 2).

Case 1. In Equation (1) when $x=t$, the function $k(x, t)$ satisfies the Lipchitz condition and the function $u(x)$ is continuous, then Equation (1) is equivalent to
Equation (6), then the function $u(x)=f(x)$ which gives us the exact solution.

Case 2. In this case we don't have singularities, and Equation (1) is equivalent to Equation (6) and $H(x, t)=k(x, t)|x-t|^{\alpha}$. Subtracting Equation (7) from (6) and applying the norm, we have

$$
\begin{aligned}
& \|e(x)\|=\left\|\sum_{p} a_{p} \varphi_{j, p}(t)-u(t)\right\| \\
& =\left\|\int_{0}^{1} H(x, t)\left(\sum_{p} a_{p} \varphi_{j, p}(t)-u(t)\right) \mathrm{d} t\right\| \\
& \leq\left\|\int_{0}^{1} H(x, t) \mathrm{d} t\right\|\left\|\int_{0}^{1}\left(\sum_{p} a_{p} \varphi_{j, p}(t)-u(t)\right) \mathrm{d} t\right\|
\end{aligned}
$$

The unknown function $u(t)$ can be interpolated using Coiflet such that

$$
u(t) \approx u^{j}(t)=\sum_{p} u\left(\frac{p}{2^{j}}\right) \varphi_{j, p}(t) .
$$

If we add and subtract Equation (9) to (8), then Equation (8) becomes:

$$
\begin{aligned}
& \|e\| \leq c_{1}\left(\left\|\int_{0}^{1}\left(\sum_{p} u\left(\frac{p}{2^{j}}\right) \phi_{j, p}(t)-u(t)\right) \mathrm{d} t\right\|\right. \\
& \left.+\left\|\int_{0}^{1}\left(\sum_{p} u\left(\frac{p}{2^{j}}\right)-a_{p}\right) \phi_{j, p}(t) \mathrm{d} t\right\|\right)
\end{aligned}
$$

Notice that $\sum_{p} u\left(\frac{p}{2^{j}}\right)-a_{p}$ is finite, then let

$c_{2}=\sum_{p} u\left(\frac{p}{2^{j}}\right)-a_{p}$

and by using Equation (2),

$$
\left\|u-u^{j}\right\|_{L^{2}(\Omega)} \leq c_{0}\left\|u^{(N)}\right\|_{\infty}\left(\frac{1}{2^{j}}\right)^{N-1}
$$

Equation (10) becomes

$$
\|e\| \leq c_{1}\left(c_{0}\left\|u^{(N)}\right\|\left(\frac{1}{2}\right)^{N-1}+c_{2}\left(\frac{1}{2}\right)^{j}\right)=c\left(\frac{1}{2}\right)^{j}
$$

for some constant $c$ which is absorbed from the above inequality.

\section{Numerical Examples}

In the following examples we are solving singular Fredholm integral equation of the second kind by using Coiflet of order 5 and calculate errors between the exact and numerical solutions at level $j=-10$. The errors are shown in Table 1.

\section{Example 1.}

We solve the singular integral equation 
Table 1. The absolute error for Examples 1 and 2.

\begin{tabular}{ccc}
\hline$X$ & Error for Example 1 & Error for Example 2 \\
\hline 0.1 & $3.46054 \mathrm{E}-10$ & $1.98116 \mathrm{E}-7$ \\
0.2 & $3.01383 \mathrm{E}-11$ & $1.16379 \mathrm{E}-7$ \\
0.3 & $1.95466 \mathrm{E}-10$ & $9.29447 \mathrm{E}-8$ \\
0.4 & $3.30836 \mathrm{E}-10$ & $8.37863 \mathrm{E}-8$ \\
0.5 & $3.76019 \mathrm{E}-10$ & $6.1996 \mathrm{E}-8$ \\
0.6 & $3.30948 \mathrm{E}-10$ & $8.37863 \mathrm{E}-8$ \\
0.7 & $1.95553 \mathrm{E}-10$ & $9.29447 \mathrm{E}-8$ \\
0.8 & $3.01101 \mathrm{E}-11$ & $1.16379 \mathrm{E}-7$ \\
0.9 & $3.46045 \mathrm{E}-10$ & $1.98116 \mathrm{E}-7$ \\
\hline
\end{tabular}

$$
u(x)=f(x)+\int_{0}^{1} \frac{1}{10}|x-t|^{-\frac{1}{3}} u(t) \mathrm{d} t
$$

with

$$
\begin{aligned}
f(x)= & x^{2}\left(1-x^{2}\right)-\frac{27}{30800}\left(x^{8 / 3}\left(54 x^{2}-126 x+77\right)\right) \\
& +(1-x)^{8 / 3}\left(54 x^{2}+18 x+5\right)
\end{aligned}
$$

and the exact solution is $u(x)=x^{2}(1-x)^{2}$.

\section{Example 2.}

We consider the following singular integral equation

$$
\begin{aligned}
& \frac{3 \sqrt{2}}{4} u(x)-\int_{0}^{1}|x-t|^{-1 / 2} u(t) \mathrm{d} t \\
& =3\left(x(1-x)^{3 / 4}-\frac{3}{8} \pi(1+4 x(1-x))\right)
\end{aligned}
$$

with the exact solution $2 \sqrt{2}(x(1-x))^{3 / 4}$

\section{Conclusion}

We apply our method to the same examples shown in [3]. Table 1 indicates that our solutions have better accuracy than the solutions obtained in [3]. Our method is robust and efficient. There are other questions such as finding solutions at different levels of subspaces and solving nonlinear integral equations which will be our next research projects.

\section{REFERENCES}

[1] E. Lin and Y. Al-Jarrah, "A Wavelet Based Method for the Solution of Fredholm Integral Equations," American Journals of Computational Mathematics, Vol. 2, No. 2, 2012, pp. 114-117.

[2] H. Derili and S. Sohrabi, "Numerical Solution of Singular Integral Equations Using Orthogonal Functions," Mathematical Science, Vol. 2, No. 3, 2008, pp. 261-272.

[3] K. Maleknejad, M. Nosrati and E. Najafi, "Wavelet Galerkin Method for Solving Singular Integral Equations," Computational \& Applied Mathematics, Vol. 31, No. 2, 2012, pp. 1-14.

[4] E. Hermandez and G. Weiss, "A First Course on Wavelets," CRC Press, 2010.

[5] E. Lin and X. Zhou, "Coiflet Interpolation and Approximation Solutions of Elliptic Partial Differential Equations," Numerical Methods for Partial Differential Equations, Vol. 13, No. 4, 1997, pp. 303-320. 